\title{
Neonikotinoide - Wie eine Wirkstoffgruppe die Entwicklung einer In-vitro-Larventestmethode für Honigbienen fördert
}

\author{
Rolf Forster
}

Published online: 27 March 2014

(C) Bundesamt für Verbraucherschutz und Lebensmittelsicherheit (BVL) 2014

Zum Jahresbeginn 2003 beklagten die deutschen Imker massive Verluste von Bienenvölkern. Die Erhebungen in den Imkereien offenbarten durchschnittliche Verluste von etwa $30 \%$. Für viele war der Fall klar: Die Saatgutbehandlung von Raps, aber auch Mais, mit Neonikotinoiden sollte die Bienenvölker über vergifteten Nektar und Pollen derartig geschwächt haben, dass diese den Winter nicht überlebten. In Frankreich wurde seitens der Imkerschaft die Saatgutbehandlung von Sonnenblumen und Mais mit dem Wirkstoff Imidacloprid für die dort seit den 90er Jahren berichteten Bienenverluste verantwortlich gemacht. Diese Annahme wurde durch den auf fachlicher Ebene höchst umstrittenen "CST-Bericht" (Comite Scientifique et Technique de l'Etude Multifactorielle des Troubles des Arbeilles, CST 2003) gestützt. Zudem wurde das Fehlen jeglicher Daten zur Toxizität des Wirkstoffes für Bienenlarven als Datenlücke bemängelt. Tatsache war, dass die Völkerverluste aus dem Winter 2002/ 2003 trotz langjähriger Anwendung von Pflanzenschutzmitteln mit dem Wirkstoff Imidacloprid in Deutschland erstmalig beobachtet wurden. Ferner, dass der Wirkstoff Imidacloprid seit der Zulassung 1993 in keiner Bienen- oder Pflanzenprobe, die bei der Untersuchungsstelle für Bienenvergiftungen in der Biologischen Bundesanstalt für Land- und Forstwirtschaft zur Aufklärung von Bienenschäden untersucht worden waren, nachgewiesen werden

Dr. R. Forster $(\bowtie)$

Bundesamt für Verbraucherschutz und

Lebensmittelsicherheit, Messeweg 11/12,

38104 Brunswick, Germany

e-mail: rolf.forster@bvl.bund.de konnte. Auf einem BVL-Symposium im Jahr 2004 wurde resümiert, dass aufgrund aller vorhandenen wissenschaftlichen Erkenntnisse die behaupteten Pflanzenschutzmittelwirkungen nicht ursächlich mit dem Bienensterben des Winters 2002/2003 in Verbindung gebracht werden konnten. Vielmehr wurde die Hypothese formuliert, dass der Gesamtkomplex der die Honigbiene beeinflussenden Stressoren von zentraler Bedeutung sein könnte. Hierzu gehören insbesondere die Einflüsse von Witterung, Trachtangebot, Bekämpfung der Varroamilbe, die Varroamilbe selbst sowie die von ihr übertragenen Krankheiten und andere Bienenkrankheiten und nur in Ausnahmefällen die Anwendung von Pflanzenschutzmitteln (Forster et al. 2005). Eine Einschätzung, die im Rahmen des bundesweiten Bienenmonitorings, das infolge des Symposiums begründet wurde, bestätigt werden sollte (Genersch et al. 2010). Allerdings wurde auch bemängelt, dass die Wirkung von Pflanzenschutzmitteln auf die Bienenbrut nicht in Laborstudien untersucht worden war und festgestellt, dass Bedarf an der Entwicklung einer entsprechenden Untersuchungsmethode bestehe. Bis dato waren jedoch verschiedene Versuche, ein praxisreifes In-vitro-Testverfahren für Bienenlarven zu entwickeln, gescheitert. Erst im Jahr 2005 konnten französische Wissenschaftler eine geeignete Methode präsentieren (Aupinel et al. 2005).

Im Frühjahr 2008 ereignete sich dann der bis heute größte Vergiftungsfall durch Neonikotinoide in Deutschland: Aus Süddeutschland wurden Bienenvergiftungen gemeldet, bei denen nach den Erhebungen des Landes Baden-Württemberg etwa 11.500 Völker geschädigt wurden. Bei diesen wurden gravierende Flug- und Stockbienenverluste, aber auch 
Brutschäden festgestellt. Sofort nach Bekanntwerden der Vorfälle begann eine intensive Suche nach den Ursachen. Bereits eine Woche nach diesen Bienenvergiftungen wurde vermutet, dass mit einem Neonikotinoid behandeltes Maissaatgut die Ursache für diese Vergiftungen war, was durch die Analysen von Bienen- und Pflanzenproben sowie von einzelnen Saatgutchargen bestätigt werden konnte. Der nachgewiesene Wirkstoff Clothianidin stammte offensichtlich von behandeltem Maissaatgut, bei dem der Wirkstoff nicht ausreichend an den Körnern anhaftete, so dass es zu einem starken Abrieb und einer Anhäufung von Stäuben in einigen Saatgutpartien kam. In der Oberrheinebene wurden zudem pneumatische Sägeräte mit Saugluftsystemen verwendet, die aufgrund ihrer speziellen Konstruktion den Clothianidin-haltigen Staub über die Abluftführung direkt in die Luft abgaben und so auch auf blühende Pflanzen verteilten (Pistorius et al. 2008). Noch vor der vollständigen Aufklärung der Vorfälle ordnete das BVL das Ruhen der Zulassung für eine Reihe von insektiziden Saatgutbehandlungsmitteln an. Kurz darauf folgte das Verbot des Bundesministeriums für Ernährung, Landwirtschaft und Verbraucherschutz für das Inverkehrbringen und die Aussaat von mit Neonikotinoiden behandeltem Maissaatgut. Aufgrund der berichteten Brutschäden vereinbarte das BVL mit dem LAVES Institut für Bienenkunde darüber hinaus ein Projekt zur Etablierung und Validierung eines In-vitro-Larventests. Zusammen mit dem Julius Kühn-Institut, dem Bundesverband der Deutschen Pflanzenzüchter und dem Verband Deutscher Maschinen- und Anlagenbau konnten zudem in den Folgejahren technische Lösungen zur Minimierung der Staubemission erarbeitet werden.

Anfang 2009 alarmierten die Experimente italienischer Bienenwissenschaftler (Girolami et al. 2009) erneut die Öffentlichkeit: Das Guttationswasser behandelter Maispflanzen wies Neonikotinoidkonzentrationen auf, die als hochgradig bienentoxisch $\mathrm{zu}$ bewerten waren. Untersuchungen des Julius Kühn-Instituts zeigten zudem, dass Guttation auch bei einer Vielzahl weiterer Pflanzenarten wie Raps und Zuckerrüber, aber auch Ackerunkräutern und Gräsern beobachtet werden kann. Während frühere Untersuchungen belegt hatten, dass die nachgewiesenen Wirkstoffkonzentrationen von Neonikotinoiden in Nektar und Pollen durchweg im Bereich der Nachweisgrenze und unterhalb der für Honigbienen als relevant bewerteten Schwellenwerte liegen, ergaben Rückstandsanalysen in den Guttationstropfen Wirkstoffgehalte von teilweise mehr als
$1 \mathrm{mg}$ Wirkstoff pro Liter. Es wurde auch festgestellt, dass die Kulturpflanzen in der Regel während der Jungpflanzenentwicklung guttieren und dass in dieser Phase die Konzentration von Wirkstoffen über mehrere Wochen sehr hoch sein kann. Folglich stellte sich die Frage nach einem möglichen Eintrag von Guttationswasser in die Bienenvölker und der Exposition der verschiedenen Entwicklungsstadien einschließlich der Bienenlarven. Gezielte Freilanduntersuchungen aus dem Jahr 2010 ergaben, dass bei einer Aufstellung der Völker unmittelbar an einem Maisfeld zwar eine geringfügige Erhöhung des natürlichen Totenfalls der Bienen nicht ausgeschlossen werden kann. In anderen Untersuchungen, in denen die Bienenvölker in einem Abstand von nur wenigen Metern zum Maisfeld aufgestellt wurden und denen alternative Wasserquellen zur Verfügung standen, wurden diese Auswirkungen jedoch nicht festgestellt.

Eine weitere kontrovers geführte Diskussion betraf die Annahme, dass für die Wirkstoffe aus der Gruppe der Neonikotinoide die Habersche Regel anwendbar sei (Tennekes 2010). Diese besagt, dass das Produkt aus Menge und Dauer einer Giftgabe einer konstanten biologischen Wirkung entspricht und folglich selbst kleinste Mengen bei dauerhafter Exposition zu Schäden führen. Dem wird entgegengehalten, dass die Habersche Regel nur bei irreversiblen Wirkungen von Summationsgiften wie beispielsweise krebserregenden Stoffen anwendbar ist und für die Wirkung von Neonikotinoiden nicht belegt ist. Um diese Frage auch experimentell abklären zu können, war jedoch zunächst die Erweiterung des akuten Larventests zu einem chronischen Test erforderlich. Entsprechende Untersuchungen wurden im gleichen Jahr am LAVES Institut für Bienenkunde begonnen (Lüken et al. 2012).

Im Frühjahr 2012 erschienen zwei Studien, in denen bienenschädigende Auswirkungen subletaler Dosen von Neonikotinoiden wie Imidacloprid und Thiamethoxam auf bestäubende Insekten [Honigbienen (Whitehorn et al. 2012) und Hummeln (Henry et al. 2012)] berichtet wurden: Diese beeinflussten den Orientierungssinn, das Koloniewachstum von Hummelvölkern sowie die Bildung neuer Hummelköniginnen. Über diese Experimente wurde in der Folge ein intensiver wissenschaftlicher Disput geführt und festgestellt, dass die Untersuchungsergebnisse zwar auf ein potenzielles Risiko durch Neonikotinoide für Hummeln und Honigbienen unter bestimmten Expositionsbedingungen hinweisen, diese aber vor dem Hintergrund der wissenschaftlichen Erkenntnisse hinsichtlich der 
Expositionsdauer bzw. -höhe als wenig realistisch zu bewerten waren.

Mitte 2013 wurde von der Kommission die Durchführungsverordnung (EU) Nr. 485/2013 (EU 2013) in Kraft gesetzt, in der weitreichende Restriktionen bezüglich der Anwendung der Wirkstoffe Imidacloprid, Clothianidin und Thiamethoxam festgelegt wurden. Die EU-Kommission hatte ferner angekündigt, sie werde innerhalb von zwei Jahren eine Überprüfung neuer wissenschaftlicher Erkenntnisse anstrengen, die ihr bis dahin vorgelegt worden sind. Ende Juli 2013 konnte die OECD die Erstellung einer Testrichtlinie für den In-vitro-Larventest vermelden (OECD 2013) - ein chronischer Test ist in Vorbereitung.

Der in dieser Ausgabe des JVL erscheinende Bericht des LAVES Institut für Bienenkunde (Lüken et al. 2014a, b) leistet einen wichtigen Beitrag für eine erfolgreiche Durchführung neuer In-vitro-Larventests und die Weiterentwicklung auch des chronischen In-vitro-Larventests. Die intensiven wissenschaftlichen Untersuchungen zum Verständnis der Neonikotinoide in Pflanzenschutzmitteln in Bezug auf den Schutz der Bienen haben damit wesentlich dazu beigetragen, die genannte Testmethode zu entwickeln und zu validieren, was letztlich auch die Prüfung und Bewertung anderer Pflanzenschutzmittel qualitativ verbessern wird.

\section{Literatur}

Aupinel P, Fortini D, Dufour H, Tasei JN, Michaud B, Odoux JF, Pham Delègue $\mathrm{MH}$ (2005) Improvement of artificial feeding in a standard in vitro method for rearing Apis mellifera larvae. Bull Insectol 58(2):107-111

Comite Scientifique et Technique de l'Etude Multifactorielle des Troubles des Arbeilles (CST) (2003) Imidaclopride utilise en enrobage de semences (Gaucho) et troubles des arbeilles-Rapport final. Ministere de l'Agriculture de la Peche et des Affaires Rurales, p 221

EU (2013) Durchführungsverordnung (EU) Nr. 485/2013 der Kommission vom 24. Mai 2013 zur Änderung der Durchführungsverordnung (EU) Nr. 540/2011 hinsichtlich der Bedingungen für die Genehmigung der Wirkstoffe Clothianidin, Thiamethoxam und Imidacloprid sowie des Verbots der Anwendung und des Verkaufs von Saatgut, das mit diese Wirkstoffe enthaltenden Pflanzenschutzmitteln behandelt wurde. Amtsblatt der Europäischen Union L 139/12, 25.5.2013

Forster R, Bode E, Brasse D (2005) Das "Bienensterben" im Winter 2002/2003 in Deutschland-zum Stand der wissenschaftlichen Erkenntnisse. Herausgeber: Bundesamt für Verbraucherschutz und Lebensmittelsicherheit. Braunschweig, 125 Seiten

Genersch E, von der Ohe W, Kaatz H, Schroeder A, Otten C, Büchler R, Berg S, Ritter W, Mühlen W, Gisder S, Meixner M, Liebig G, Rosenkranz P (2010) Das Deutsche BienenMonitoring-Projekt: Eine Langzeitstudie zur Untersuchung periodisch auftretender hoher Winterverluste bei Honigbienenvölkern. Apidologie INRA/DIB-AGIB/EDP Sciences

Girolami V, Mazzon L, Squartini A, Mori N, Marzaro M, Di Bernardo A, Greatti M, Giorio C, Tapparo A (2009) Translocation of neonicotinoid insecticides from coated seeds to seedling guttation drops: a novel way of intoxication for bees. J Econ Entomol 102(5):1808-1815. doi:10.1603/029.102.0511

Henry M, Beguin M, Requier F, Rollin O, Odoux J-F, Aupinel P, Aptel J, Tchamitchian S, Decourtye A (2012) A common pesticide decreases foraging success and survival in honey bees. Science 336(6079):348-350

Lüken DJ, Janke M, Lienau F-W, von der Ohe W, Forster R (2012) Weiterentwicklung einer Methode zur Bienenbruthaltung unter Laborbedingungen. J Verbr Lebensm 7(2):141-145

Lüken DJ, Janke M, Lienau FW, Gerdel K, von der Ohe W, Forster R (2014a) Optimierung eines In-vitro-Larventests zur Prüfung von Pflanzenschutzmittelwirkungen auf die Bienenbrut (Apis mellifera L.)-Abschlussbericht über die Forschungsprojekte im Zeitraum 15.07.2008-31.03.2012. J Verbrauch Lebensm 9(2). doi:10.1007/s00003-013-0850-z

Lüken DJ, Janke M, Lienau FW, Gerdel K, von der Ohe W, Forster R (2014b) Optimisation of an in vitro larvae test to test the effects of plant protection products on honeybee brood (Apis mellifera L.)-final report on research projects (duration: 15 July 2008 to 31 March 2012). J Verbrauch Lebensm 9(2). doi:10.1007/s00003-014-0865-0

OECD (2013) Honey bee (Apis mellifera) Larval toxicity test, single exposure. OECD guidelines for the testing of chemicals no 237, OECD, Paris

Pistorius J, Bischoff G, Glas M, Heimbach U, Trenkle A, Stähler M (2008) Maisanbau und Bienenvergiftungen-Hintergründe und Fakten. Mitt Julius Kühn-Institut 417:206

Tennekes HA (2010) The systemic insecticides: a disaster in the making. EBook or Hardcover from http://www. adisasterinthemaking.com or in the UK from Northern Bee Books; sales@recordermail.demon.co.uk

Whitehorn PR, O'Connor S, Wackers FL, Goulson D (2012) Neonicotinoid pesticide reduces bumble bee colony growth and queen production. Science 336(6079):351-352 